\title{
Estimating relationship between the time over threshold and energy loss by photons in plastic scintillators used in the J-PET scanner
}

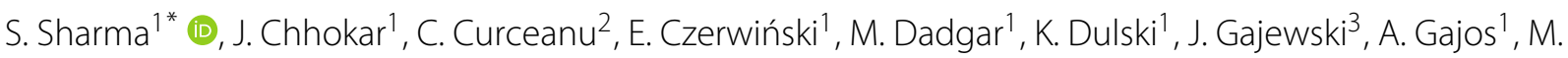 \\ Gorgol $^{4}$, N. Gupta-Sharma ${ }^{4}$, R. Del Grande², B.C. Hiesmayr ${ }^{5}$, B. Jasińska ${ }^{4}$, K. Kacprzak , Ł. Kapłon , H. \\ Karimi ${ }^{1}$, D. Kisielewska ${ }^{1}$, K. Klimaszewski ${ }^{6}$, G. Korcyl ${ }^{1}$, P. Kowalski ${ }^{6}$, T. Kozik ${ }^{1}$, N. Krawczyk ${ }^{1}$, W. Krzemień ${ }^{7}$, E.

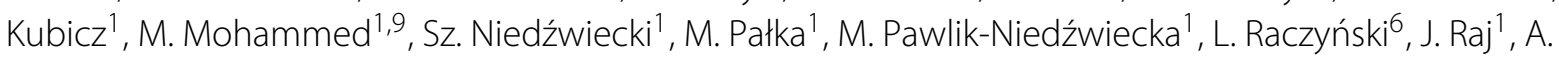 \\ Ruciński ${ }^{3}$, Shivani ${ }^{1}$, R.Y. Shopa ${ }^{6}$, M. Silarski ${ }^{1}$, M. Skurzok ${ }^{1}, 2$, E.Ł. Stępień ${ }^{1}$, W. Wiślicki ${ }^{7}$, B. Zgardzińska ${ }^{4}$ \\ and P. Moskal ${ }^{1}$
}

*Correspondence: sushil.sharma@uj.edu.pl ${ }^{1}$ Faculty of Physics, Astronomy and Applied Computer Science, Jagiellonian University, prof. Stanisława Łojasiewicza 11, 30-348 Cracow, Poland

Full list of author information is available at the end of the article

\begin{abstract}
Purpose: The time-over-threshold (TOT) technique is being used widely due to its implications in developing the multi-channel readouts, mainly when fast signal processing is required. Using the TOT technique, as a measure of energy loss instead of charge integration methods, significantly reduces the signal readout costs by combining the time and energy information. Therefore, this approach can potentially be utilized in J-PET tomograph which is built from plastic scintillators characterized by fast light signals. The drawback in adopting this technique lies in the non-linear correlation between input energy loss and TOT of the signal. The main motivation behind this work is to develop the relationship between TOT and energy loss and validate it by the J-PET tomograph setup.
\end{abstract}

Methods: The experiment was performed using a ${ }^{22} \mathrm{Na}$ beta emitter source placed in the center of the J-PET tomograph. This isotope produces photons of two different energies: $511 \mathrm{keV}$ photons from the positron annihilation (direct annihilation or through the formation of a para-positronium atom or pick-off process of ortho-positronium atoms) and a $1275 \mathrm{keV}$ prompt photon. This allows the study of the correlation between TOT values and energy loss for energy ranges up to $1000 \mathrm{keV}$. Since the photon interacts predominantly via Compton scattering inside the plastic scintillator, there is no direct information of the energy deposition. However, using the J-PET geometry, one can measure the scattering angle of the interacting photon. Since the ${ }^{22} \mathrm{Na}$ source emits photons of two different energies, it is necessary to know unambiguously the energy of incident photons and their

(Continued on next page) (c) The Author(s). 2020 Open Access This article is licensed under a Creative Commons Attribution 4.0 International License, which permits use, sharing, adaptation, distribution and reproduction in any medium or format, as long as you give appropriate credit to the original author(s) and the source, provide a link to the Creative Commons licence, and indicate if changes were made. The images or other third party material in this article are included in the article's Creative Commons licence, unless indicated otherwise in a credit line to the material. If material is not included in the article's Creative Commons licence and your intended use is not permitted by statutory regulation or exceeds the permitted use, you will need to obtain permission directly from the copyright holder. To view a copy of this licence, visit http://creativecommons.org/licenses/by/4.0/. 
(Continued from previous page)

corresponding scattering angles in order to estimate energy deposition. In summary, this work presents a dedicated algorithm developed to tag photons of different energies and studying their scattering angles to calculate the energy deposition by the interacting photons.

Results: A new method was elaborated to measure the energy loss by photons interacting with plastic scintillators used in the J-PET tomograph. We find the relationship between the energy loss and TOT is non-linear and can be described by the functions TOT $=A 0+A 1 * \ln \left(E_{d e p}+A 2\right)+A 3 *\left(\ln \left(E_{d e p}+A 2\right)\right)^{2}$ and TOT $=A 0-A 1 *$ $A 2^{E_{\text {dep }}}$. In addition, we also introduced a theoretical model to calculate the TOT as a function of energy loss in plastic scintillators.

Conclusions: A relationship between TOT and energy loss by photons interacting inside the plastic scintillators used in J-PET scanner is established for a deposited energy range of 100-1000 keV.

Keywords: Positron emission tomography, Time over threshold, Positronium atoms, Medical imaging

\section{Background}

The time-over-threshold (TOT) technique was first time introduced by Nygren and Millaud [1] and proved to be an excellent solution for multi-channel readouts [2]. In the TOT method, one measures the signal pulse width at a selected threshold, which can be used to estimate the signal's charge. For energy deposition estimation, this method is less precise in comparison to the charge integration method. However, it reduces the readout costs by using only the time to digital converter (TDC), herewith combining both timing and energy information. The application of the TOT method for the energy loss determination may be of particular advantage in the newly developed Jagiellonian-positron emission tomograph (J-PET) [3, 4] which is based on plastic scintillators characterized by fast light signals with rise and decay times of the order of $\approx 1 \mathrm{~ns}[5,6]$ and thus being about two orders of magnitude shorter than signals from crystals used in the current PET devices [7-9]. Therefore, the application of the TOT method for PET tomographs built from plastic scintillators will enable fast signal processing and significantly reducing signal acquisition dead time with respect to the crystal based tomographs. Despite many advantages like compactness of signals readout and low power consumption, the TOT technique confronts the challenge in terms of non-linear input energy to pulse width conversion [10-12]. It has been reported that using multiple fixed triggering thresholds $[13,14]$ or dynamic threshold levels $[15,16]$ for estimating the TOT values alleviates the problem of non-linearity to a significant extent.

In PET applications, the precise determination of the photon's interaction position, hit time, and energy loss is crucial. Currently, in PET scanners, crystal scintillators are used and they enable the determination of the energy of the interacting photons as they undergo photoelectric effect [17-19]. In contrast to crystals in plastic scintillators, the incident photon interacts via Compton scattering depositing only part of its energy. Thus, there is no direct information on the energy deposition of the interacting photon.

TOT studies with plastic scintillators are very scarce [20]. In the recent work of Ashrafi and Gol [21], the energy calibration of a plastic scintillator based on Compton scattering and observing the light yield by using various monochromatic photon sources was 
reported. In this work, we take advantage of the multilayer cylindrical acceptance of the J-PET scanner [3,22] which allows us to determine the direction of the photon before and after the scattering which in turn gives us access to its scattering angle $[4,23]$. Thus, knowing the incident energy and the measured scattering angle of the initial photon, the deposited energy in the photon's interaction can be calculated [24].

For the present study, a ${ }^{22} \mathrm{Na}$ source was used which emits photons of two different energies: $511 \mathrm{keV}$ (annihilation) and $1275 \mathrm{keV}$ (prompt). The $511 \mathrm{keV}$ photons originate from the direct electron-positron annihilation, from the decay of para-positronium, and from the annihilation of ortho-positronium atoms via the pick-off processes [25-27]. Since the aim of the study is to establish the relationship between TOT and energy loss, we need to develop an algorithm capable to clearly distinguish between the annihilation and prompt photons as well as a method of proper correspondence of the measured signals to the initial and scattered photons. To achieve this, we performed studies based on the assumptions that $511 \mathrm{keV}$ photons emitted in the annihilation of $\mathrm{e}^{+} e^{-}$are always back-to-back due to momentum conservation. Secondly, to identify the prompt photons, we take advantage of the decay of long-lived ortho-positronium atoms [28] which in the XAD-4 porous polymer [29] on the average decay about $90 \mathrm{~ns}$ after the emission of a prompt photon. In this article, the presented algorithm allows us for establishing a relationship between TOT and energy deposition in plastic scintillators, a step further towards medical imaging with low cost PET devices.

\section{Methods}

The Jagiellonian-positron emission tomograph (J-PET) consists currently of 192 plastic scintillators made of EJ-230 material. A single detection module is composed of a scintillator with dimension $0.7 \times 1.9 \times 50 \mathrm{~cm}^{3}$ connected with a R9800 Hamamatsu photomultiplier on each side [30,31]. The detection modules are axially arranged in three layers: the first and second layer are composed of 48 modules whereas the third layer is the arrangement of 96 modules. Layers are not overlaying and have a diameter of $85 \mathrm{~cm}$, $93.5 \mathrm{~cm}$, and $115 \mathrm{~cm}$, respectively. The front view of three layer prototype of the J-PET scanner is shown in Fig. 1a whereas Fig. 1b exhibits its cross section. A Multi-Voltage Threshold mezzanine (MVT) board is used to probe signals at four fixed thresholds (80, 160,240 , and $320 \mathrm{mV}$ ) in the voltage domain (within the accuracy of $20 \mathrm{ps} R \mathrm{RS}$ ) [32] to achieve the good resolution of determining the hit time and place of interaction of photon inside the scintillator [3,31,33-35]. Using MVT boards connected to readouts allows us to exploit the FPGAs differential inputs as comparators. The signals from the MVT boards are sampled using TDCs implemented in the FPGA devices [36-39] whereas in standard approach, external comparator chips are used additionally [40, 41]. The data is stored in a trigger less mode with an ability to handle the data stream with rates of about 8 Gbps [42, 43]. The position of interaction along the plastic strip is calculated based on the time difference of light signals arriving at both photomultipliers. The hit-time resolution for the registration of $511 \mathrm{keV}$ photon is $\approx 155 \mathrm{ps}$ [30] whereas the spatial resolution on $\mathrm{xy}$ is $4-5 \mathrm{~mm}$ and on $z$-axis is $\approx 25 \mathrm{~mm}$ [44]. Signals from the plastic scintillators are very fast (rise time $\approx 0.5 \mathrm{~ns}$, fall time $\approx 1.8 \mathrm{~ns}$ ) [6] and are prone to much lower pile-ups with respect to crystal based detectors with an order of magnitude larger fall times [45]. Therefore, in order to avoid additional dead times in the detection system, instead of direct charge measurement only timing of the signal is used. This allows us to handle higher 


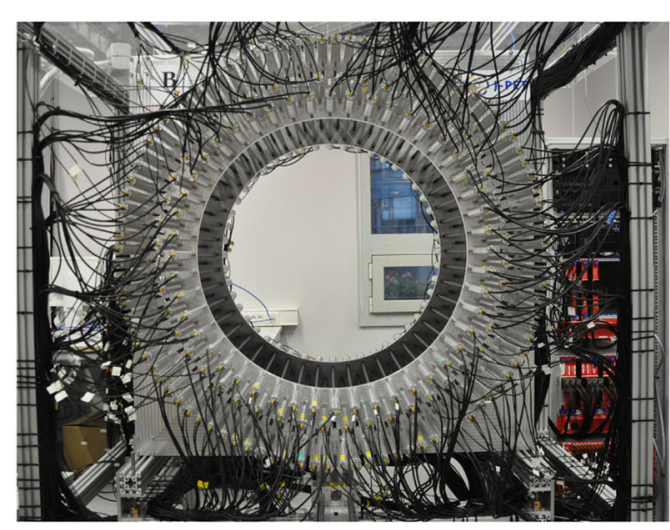

(a)

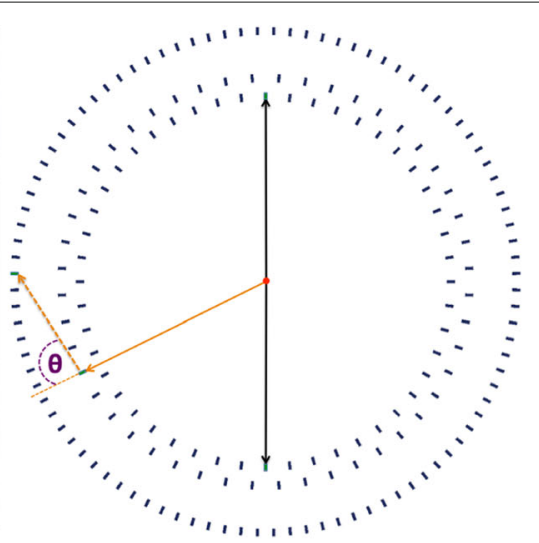

(b)

Fig. 1 a The photo of J-PET scanner with the front view (see text for description). $\mathbf{b}$ The cross section of J-PET with superimposed arrows depicting the interaction of 511 keV photons (black solid arrows) originating from positron-electron annihilation and prompt photon $1275 \mathrm{keV}$ before (orange solid arrow) and after (orange dashed arrow) scattering inside the scintillator. Red dot in the center represents the ${ }^{22} \mathrm{Na}$ source

rates of data collection. The photon's interaction in the scintillator and the arrival time of the signals in photomultipliers at each end is measured. The TOT approach is adopted instead of the charge integration method. In TOT approach, the time difference between the leading and trailing edge of signal pulse crossing the applied thresholds is measured. The schematic presentation is shown in Fig. 2. The total TOT, used as a measure of the energy deposition, is estimated as a sum of TOT values measured for all thresholds at both sides of scintillator (Eq. 1):

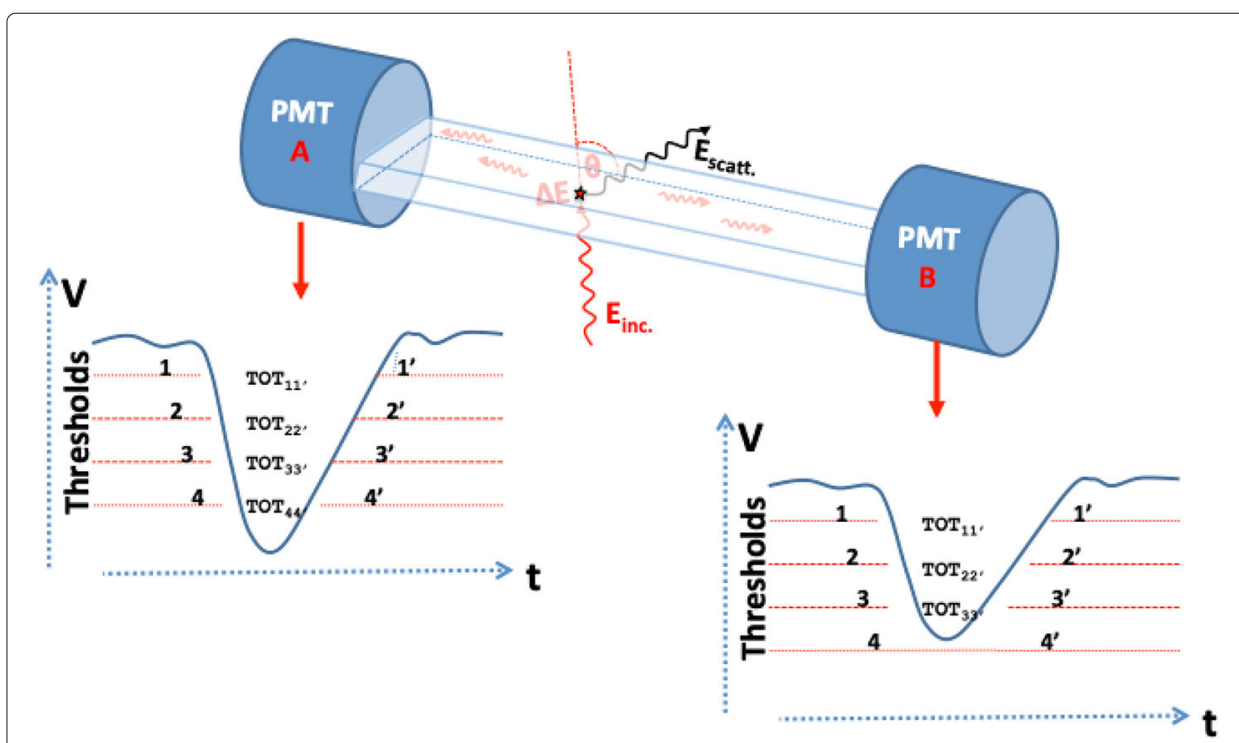

Fig. 2 Illustration of the analog signals obtained at photomultipliers as the outcome of the energy deposited by the photon in the interaction with plastic scintillator. Each signal is probed at four fixed thresholds. The TOT value is calculated at each pre-defined voltage threshold, and the sum of all four TOT values from both photomultipliers (PMTs) gives the final TOT value, Eq. 1 


$$
T O T=\sum_{P M T=A, B} \sum_{T h r_{1-4}}^{T O T_{P M T, T h r}}
$$

where $T O T_{P M T, T h r}$ is representing the sum of TOT values over all four threshold on the signals measured from both photomultipliers of each scintillator. The estimation of the charge collection using the pulse width of the signal is less precise and suffers a strongly non-linear relationship between TOT and energy deposition. The plastic scintillators are composed of hydrocarbons with low atomic $(Z)$ number; consequently, gamma photons interact in the plastic scintillators predominantly through Compton scattering and deposit only part of their energy. The information of the deposited energy is important to reduce the scatter fraction by requiring that the energy loss is larger than $200 \mathrm{keV}[3,23$, 34]. To process the measured data, a dedicated framework for offline data analysis was used $[46,47]$.

\section{Experimental set-up}

The measurements were performed with a ${ }^{22} \mathrm{Na}$ source ( $1 \mathrm{MBq}$ activity) wrapped inside a very thin Kapton foil. The experimental set-up is shown in Fig. 3. The left panel shows a picture of the J-PET tomograph with the placement of a barrel shape source holder of length $\approx 14 \mathrm{~cm}$ and diameter $\sim 3.16 \mathrm{~cm}$ (at the center). The source surrounded by the porous material was put inside a small chamber of thin layer made of aluminum and placed at the center of the holder (see the upper inset of right panel in Fig. 3). The placement of the source covered with porous material is shown at the lower right insets. In this experiment, the XAD-4 polymer was used which increases the probability of formation of positronium (Ps) atoms [29]. Using the ${ }^{22} \mathrm{Na}$ source gives a possibility to estimate the lifetime of the Ps atom by registering the annihilation photons and prompt gamma which is a feature that will allow a novel application in medical imaging. J-PET is optimized to register multiple photons simultaneously, and thus, it can be used to reconstruct the image of average lifetime of the ortho-positronium (o-Ps $\rightarrow 3 \gamma$ ) which is formed in patient body during the PET scanning process. The average lifetime of o-Ps strongly depends on the

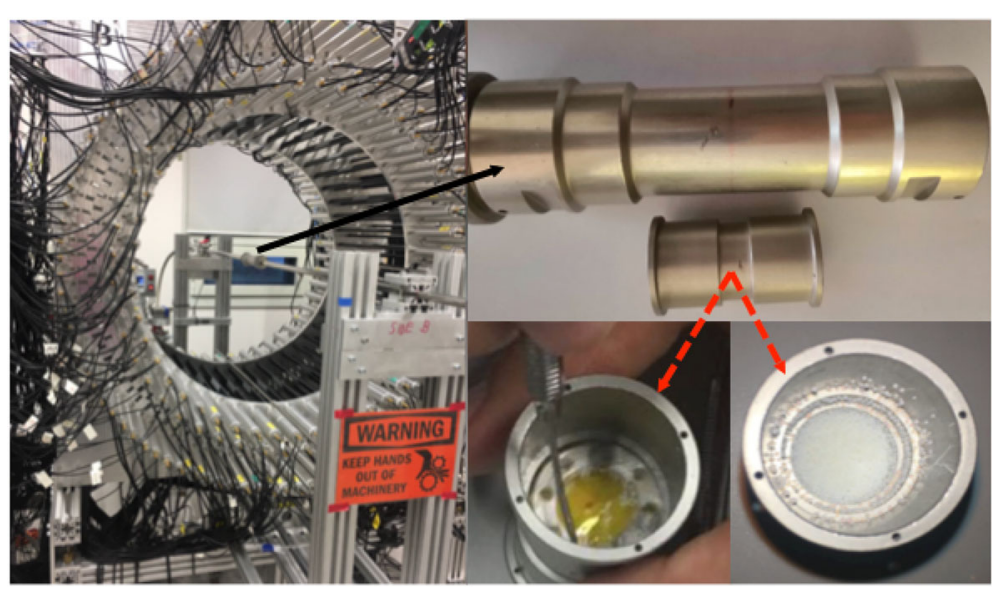

Fig. 3 Experimental set-up of the annihilation chamber with the source placed at the center of the J-PET tomograph 
free volume between atoms and thus can be used as a diagnostic indicators of human tissues [26, 27]. In the decay of ${ }^{22} \mathrm{Na}$ source (Fig. 4a), the positron is emitted leaving behind an excited state of ${ }^{22} \mathrm{Ne}$ nucleus which eventually de-excites via the emission of a prompt photon on average within the time interval of $\approx 3.7 \mathrm{ps}$. The time difference between the interaction of annihilation and prompt photons is then with a very good approximation equal to lifetime of Ps atom [26]. Gamma photons mainly interact inside the plastic scintillator via Compton scattering.

Figure $4 \mathrm{~b}$ shows the TOT spectrum for all registered photons. The clear Compton edges are visible at about $22 \mathrm{~ns}$ and $46 \mathrm{~ns}$ corresponding to $511 \mathrm{keV}$ and $1275 \mathrm{keV}$ photons, respectively. The observed enhancement of contributions for the lower values of TOT (below $10 \mathrm{~ns}$ ) is from the interaction of scattered photon and photons originating from the 3-photon decay of o-Ps atoms. It is worth to emphasize that TOT values could be used to disentangle between the annihilation and prompt photons to some extent. However, in the overlapping region for TOT values below $30 \mathrm{~ns}$, the identification would be ambiguous. Therefore, we developed a dedicated algorithm to uniquely identify the photons of energies $511 \mathrm{keV}$ and $1275 \mathrm{keV}$ irrespective of the TOT values (described in the "Events selection" section). Furthermore, the registration of initial and scattered photons allows us to determine the scattering angle $\theta$ (see Fig. 5). Knowing the energy $\left(E_{\text {inc }}\right)$ and scattering angle $(\theta)$ of the initial photon give access to energy deposition $\Delta \mathrm{E}$ inside the scintillator [24]:

$$
\Delta E=E_{\mathrm{inc}}\left[1-\frac{1}{1+\frac{E_{\mathrm{inc}}}{511 \mathrm{keV}}(1-\cos \theta)}\right]
$$

Determination of $\Delta \mathrm{E}$ and the measurement of TOT enables to establish the relation between these two variables.

In this work, events with three interactions were studied, assuring that all three hits forming the events are occurring in distinct scintillators. Furthermore, a photon interaction inside the scintillator is termed as hit. The required information $\left(\mathrm{E}_{\text {inc }}, \theta\right)$ can be extracted based on two hits only. However, the third hit allows event categorization in a way that we can differentiate the photons of different energies and conjecture their origins.

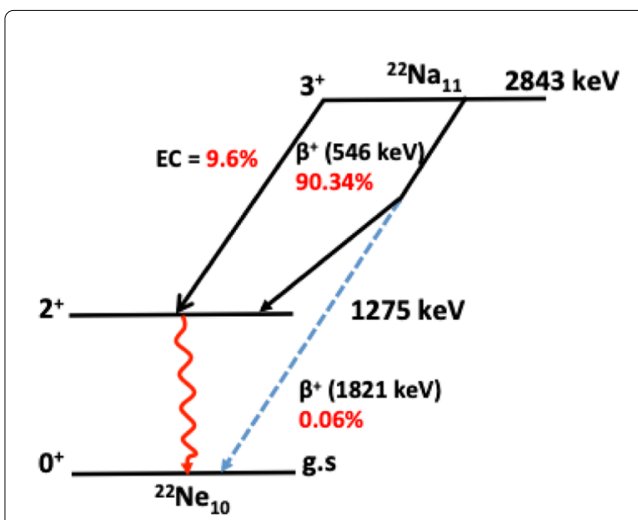

(a)

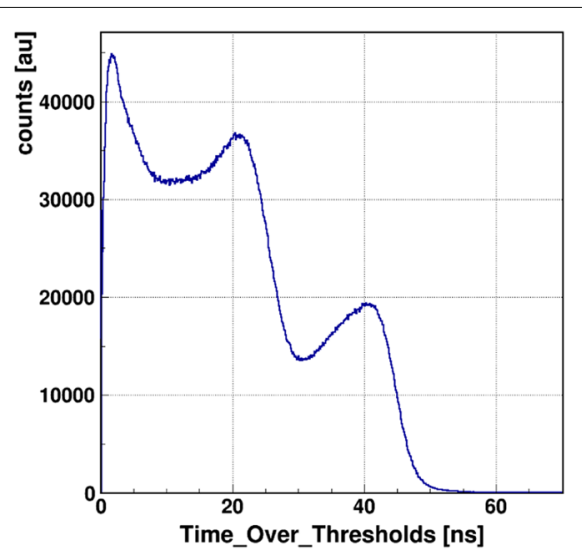

(b)

Fig. 4 a The decay scheme of the ${ }^{22} \mathrm{Na}$ source. $\mathbf{b}$ The typical TOT spectrum obtained using the ${ }^{22} \mathrm{Na}$ source 


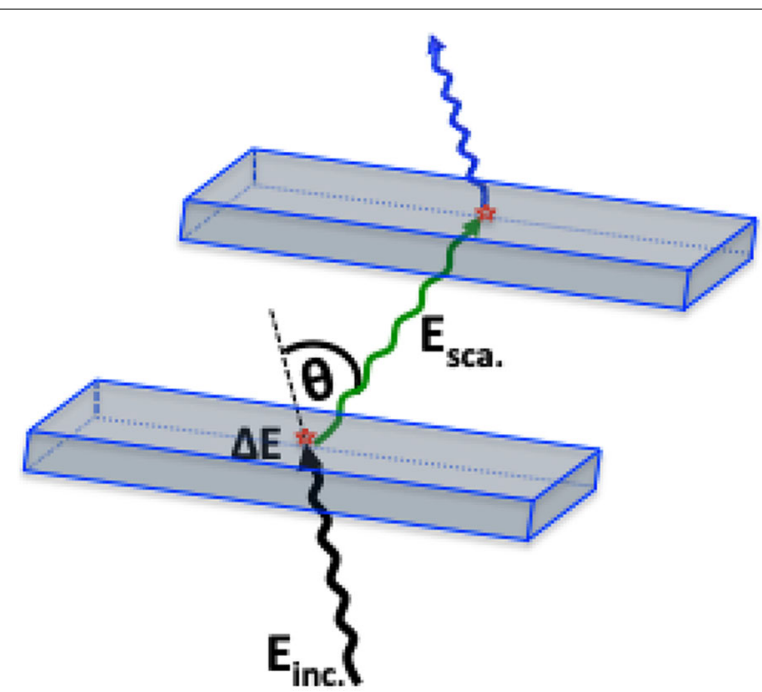

Fig. 5 The incoming and scattered photon interactions are presented with two exemplary scintillators. Knowing the positions of emission of $E_{\text {inc }}$ photon and positions of interactions of $E_{\text {inc }}$ and $E_{\text {sca }}$ photons inside the scintillators allow to estimate the scattering angle

\section{Events selection}

In this analysis, we first select the events containing three hits only. The angular correlations between the registered photons in three hit events can be used to partially identify the origin and energy of photons [48, 49]. A pictorial view of the three hits (green rectangles) and their angular correlations are presented in Fig. 6a-c. For clarity, only a few scintillators mimicking one layer of J-PET scanner are shown. The angles between three hits ordered from the smallest to the largest are $\alpha, \beta$, and $\gamma$, respectively. The measured sum of the two smallest angles vs their difference is shown in Fig. $6 \mathrm{~d}$ which shows three distinct regions: (i) $(\alpha+\beta)>180^{\circ}$, electron-positron annihilation into three photons originating from direct $e^{+} e^{-}$annihilation or from the decay of o-Ps atoms; (ii) $(\alpha+\beta)=180^{\circ}$, two back-to-back photons originating either from direct $e^{+} e^{-}$annihilation or from the decay of p-Ps atom (singlet state of Ps) or from the pick-off of o-Ps; (iii) $(\alpha+\beta)<180^{\circ}$, one or two of the hits are from scattering of initial photon or prompt photon. The coincidence time window for an event was fixed at $200 \mathrm{~ns}$ in the analysis.

\section{Identification of $511 \mathrm{keV}$ photons}

The $511 \mathrm{keV}$ photons are the outcome of $e^{+} e^{-}$annihilation into the back-to-back direction to satisfy the momentum conservation and can easily be identified in Fig. $6 \mathrm{~d}$ as a vertical line at $\alpha+\beta=180^{\circ}$. The selection of such events was done based on the condition that the sum of the two smallest angles $(\alpha+\beta)$ should be $180^{\circ}$ within the uncertainty of $2^{\circ}$. The pictorial presentation of J-PET with one layer is used to visualize the situation. In Fig. 7a, black lines represent the back-to-back photons labeled as 1 and 2 and the orange line shows the prompt photon. Scintillators with photon hits are shown as green rectangles. Hit 3 corresponds to the interaction of the scattered photon after hit 1 or hit 2 . The exact assignment of the scattering photons to its primary one is important for the proper estimation of scattering angle (Fig. 7b) which is essential for the determination of the energy deposition. For this purpose, a scatter test $\mathbf{S}$ is devised which estimates the 


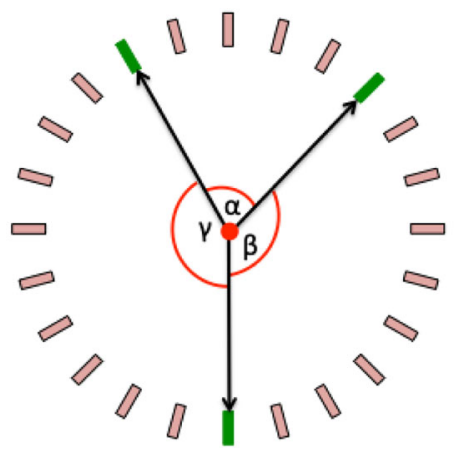

(a)

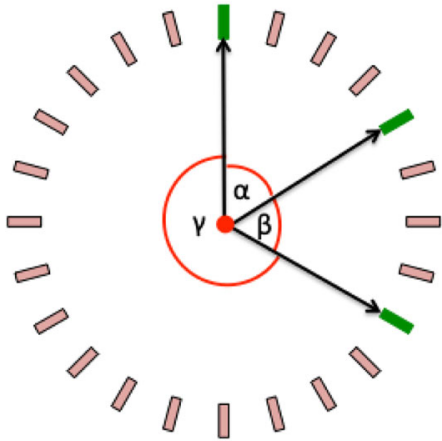

(c)

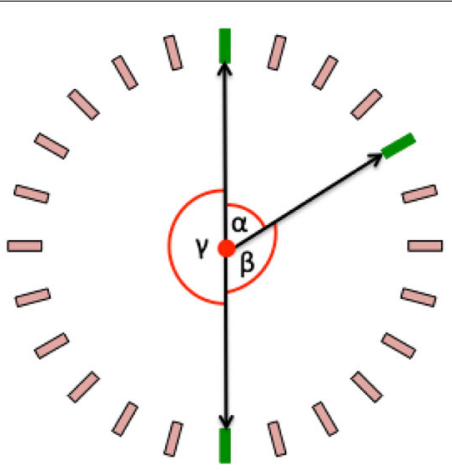

(b)

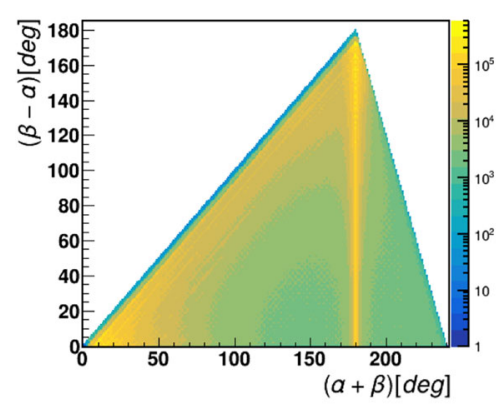

(d)

Fig. 6 a-c The three cases based on the angular correlation between the 3 hits in an event. The origin of the visualized events is explained in the text. a The case when the sum of two smallest angles is greater than $180^{\circ}$. b The case when sum is $180^{\circ}$. c When the sum of two smallest angles is less than $180^{\circ}$. $\mathbf{d}$ The measured scatter plot of the difference $(\beta-\alpha)$ vs the sum $(\alpha+\beta)$ of the two smallest angles

time difference between the measured time (time difference between two hits) and calculated time (distance between hit-positions divided by the speed of light). The $\mathbf{S}$ test was applied event-wise assuming both possibilities that the third hit might belong to the scattering photon after hit 1 or hit 2 . The results are shown in Fig. $7 \mathrm{~b}$. In case of the proper assignment, the value of $S$ should be close to zero. For the final analysis, events from the

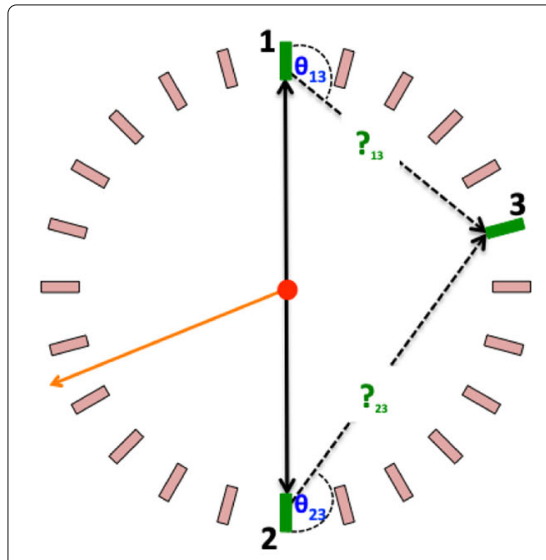

(a)

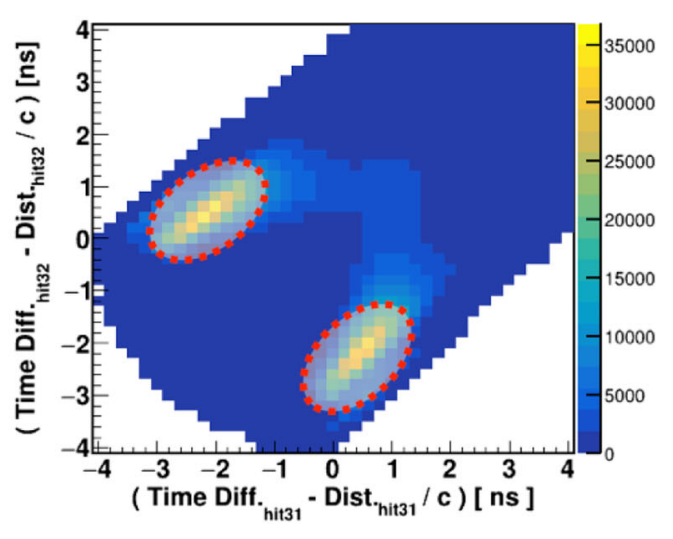

(b)

Fig. 7 a Pictorial representation of the event selection based on the algorithm used to tag the $511 \mathrm{keV}$ photons. b The encircled dotted red lines show the selected scattering events of back-to-back photons based on the scatter test $\mathbf{S}$ 
areas encircled by red-dotted lines were taken into account. With the known incident photon's energy (511 keV) and its scattering angle, the deposited energy is calculated in response to the initial hit. Thus, a one-to-one correspondence of energy deposition by $511 \mathrm{keV}$ photons and TOT values on event-wise bases is extracted. In studying $511 \mathrm{keV}$ photons, the relationship can be established only up to $340 \mathrm{keV}$ of energy deposition (the Compton edge for $511 \mathrm{keV}$ photon). So, in order to extend the relationship for higher energy deposition values, the studies with the prompt photons with energy $1275 \mathrm{keV}$ were also performed. The selection criterion for the prompt gamma is explained in the next sub-section.

\section{Identification of $1275 \mathrm{keV}$ photons and their scatterings}

The selection of prompt and its corresponding scatter photon without using an explicit cut on TOT values is not as straightforward as in the case of annihilation photons. As the first step in the selection procedure, in order to suppress events with back-to-back 511 photons, we applied cuts based on the angular correlation between the hits (Fig. 6d) such that we are not considering those events when the sum of two smallest angles lies in interval $165-185^{\circ}$. In the next step, hits are ordered according to the ascending time and only those events are selected for which the time interval between first and third hit is larger than $10 \mathrm{~ns}$. The assumed analogy is as follows: hit 1 is considered to be the prompt photon, hit 2 is the scattered photon of the prompt, and hit 3 is assumed by one of the photons originating from pick-off (Fig. 8a) or direct from the decay of orthopositronium atoms (Fig. $8 \mathrm{~b}$ ). Figure $8 \mathrm{c}$ shows the possible background in the adopted selection criterion. The time difference $(\Delta t)$ between the first and third hit is used as a key parameter. It is chosen to be much larger than the possible time difference between the scatterings of photon in the tomograph. For the present analysis, the value for $\Delta \mathrm{t}$ was fixed in the time interval (10-100 ns). For estimating the scattering angles of prompt gamma, the scattered test was applied between first and second hit. The histogram used for the scatter test is shown in Fig. 9. The acceptance time window for the true scattering of the $S$ test is chosen between -0.5 and $0.75 \mathrm{~ns}$.

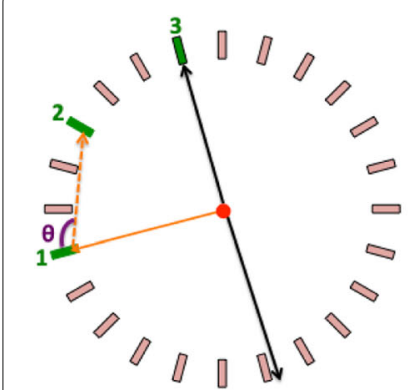

(a)

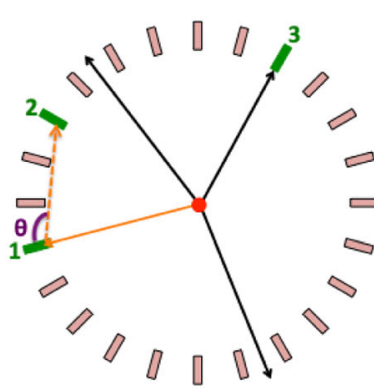

(b)

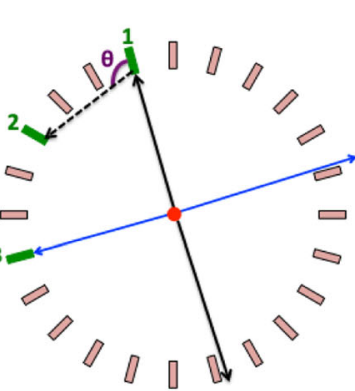

(c)

Fig. 8 Three different cases in the events selection for the $1275 \mathrm{keV}$ photons (orange arrows) based on the delayed decay of ortho-positronium atoms: a The first case shows the two annihilation photons (black arrows) from the decay of para-positronium (or ortho-positronium pick-off). $\mathbf{b}$ The photons originating from the decay of ortho-positronium state (three black arrows). c A possible source of background in the adopted procedure for the selection of prompt photon due to the accidental coincidences. The black and blue solid lines presenting the back-to-back annihilation photons might originate from two different decays 


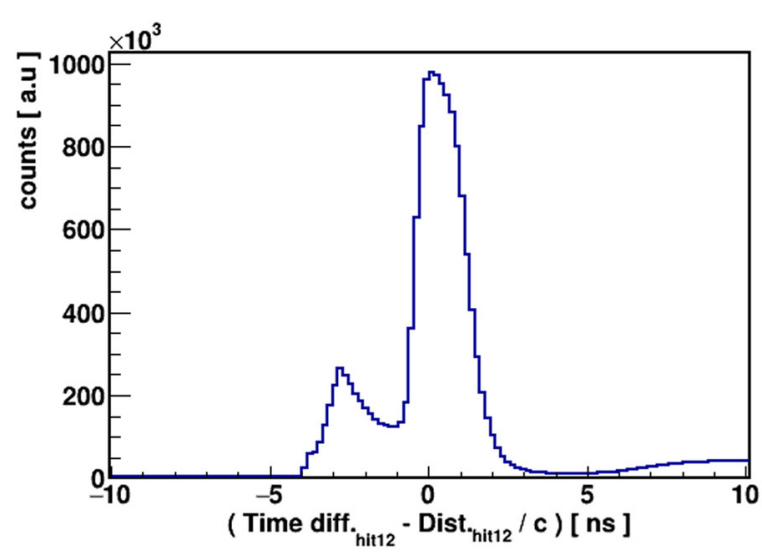

Fig. 9 Results of the $\mathbf{S}$-test calculated between hit 1 and hit 2 in event-wise manner. For the true scattering of prompt photons, the value of the $S$ test is chosen between -0.5 and $0.75 \mathrm{~ns}$. Small peak around $S=-3 \mathrm{~ns}$ is mainly due to the contribution from back-to-back annihilation photons. A slope starting at 5 ns is because of the time difference constraint between hit 1 and hit 3 (> $10 \mathrm{~ns}$ )

\section{Theoretical model}

The energy deposition by a photon inside a plastic scintillator is a function of scattering angle. Furthermore, the shape and amplitude of the signal pulse vary depending on the properties of scintillators (solvent, solute, and wavelength shifter) [50,51]. The time distribution spectra of the emitted light in plastic scintillators EJ-230 (Eljen Technology) due to an incoming photon interaction at time $\Theta$ can be estimated by the following equation $[3,50,51]$ :

$$
f(t \mid \Theta)=K \int_{\Theta}^{t}\left(e^{-\frac{t-\tau}{t_{d}}}-e^{-\frac{t-\tau}{t_{r}}}\right) \cdot e^{-\frac{(\tau-\Theta-2.5 \sigma)^{2}}{2 \sigma^{2}}} d \tau
$$

The equation is a convolution of Gaussian and exponential terms, where $\sigma$ represents the rate of energy transfer to the primary solute, whereas $t_{r}$ and $t_{d}$ denote the average time of the energy transfer to the wavelength shifter and decay time of the final light emission, respectively [51]. $K$ is the normalization factor to unity. At time $\Theta=2 \mathrm{~ns}, \mathrm{t}_{d}=1.5 \mathrm{~ns}, \mathrm{t}_{r}$ $=0.005 \mathrm{~ns}$, and $\sigma=0.2 \mathrm{~ns}$, Eq. 3 gives the time distribution spectra of emitted photons where the deposited energy is characterized by the amplitude of the signal (f). Figure 10 indicates three exemplary pulses calculated according to Eq. 3 normalized relatively to simulate signals corresponding to energy loss of $80 \mathrm{keV}$ (Fig. 10a), $500 \mathrm{keV}$ (Fig. 10b), and $900 \mathrm{keV}$ (Fig. 10c).

Each signal pulse is probed at four thresholds (f) 0.05 ( $\left.\mathrm{TOT}_{1}\right), 0.10\left(\mathrm{TOT}_{2}\right), 0.15$ $\left(\mathrm{TOT}_{3}\right)$, and $0.20\left(\mathrm{TOT}_{4}\right)$ shown by the red solid lines in Fig. 10. The total TOT value is calculated as $\mathrm{TOT}_{\text {model }}=\mathrm{N}_{0} \cdot \mathrm{TOT}_{\text {sum }}$, where $\mathrm{TOT}_{\text {sum }}=\mathrm{TOT}_{1}+\mathrm{TOT}_{2}+\mathrm{TOT}_{3}+$ $\mathrm{TOT}_{4}$ and $\mathrm{N}_{0}$ is a free parameter. The theoretical predictions will be compared with experimental results in the next section.

\section{Results}

The motivation of this work is the elaboration of a method to estimate the energy loss by incident photons interacting with plastic scintillators used in the J-PET tomography scanner. The analysis is performed with the ${ }^{22} \mathrm{Na}$ isotope because it is long lived (2.6 years half 


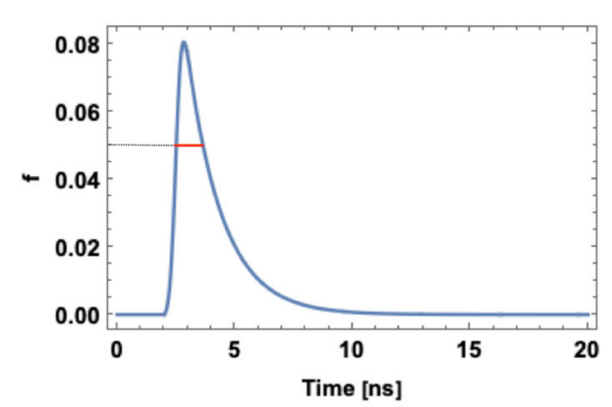

(a)

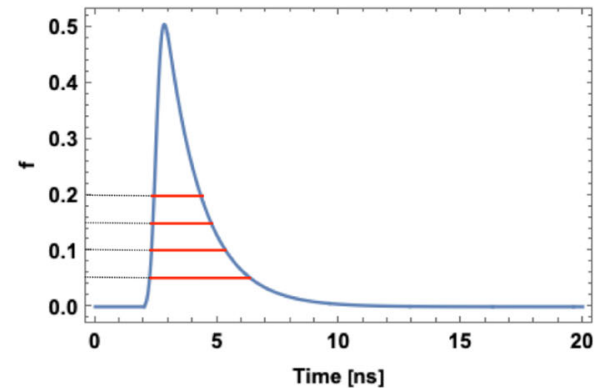

(b)

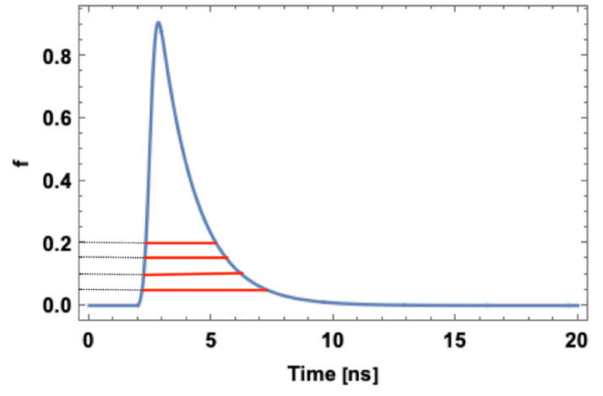

(c)

Fig. 10 Time distribution spectra of emitted light is calculated by using Eq. 3 for three cases representing signals corresponding to the energy deposition of $80 \mathrm{keV}(\mathbf{a}), 500 \mathrm{keV}(\mathbf{b})$, and $900 \mathrm{keV}$ (c)

lifetime) and is emitting positrons annihilating to $511 \mathrm{keV}$ photons as used in PET diagnostics. Since the aim of this study is to establish the relation between TOT and $\Delta \mathrm{E}$ for the plastic scintillators, in order to avoid the biasness in identification of $511 \mathrm{keV}$ (positron annihilation) and $1275 \mathrm{keV}$ (deexcitation) photons, a new method of photon identification was developed which is independent of the TOT values. After selecting the photons of different energies, their scattering angles are estimated based on the hit characteristics. With information of incident energy of photon and its scattering direction, the energy transfer to the electrons inside the scintillator can be calculated using the Compton scattering formula (Eq. 2).

In each event, hit characteristics are measured that comprise the TOT values and photon's interaction time as well as spatial coordinates. For all selected hits, the relationship between measured TOT values and the estimated energy deposition is presented in Fig. 11. An enhancement in TOT values is observed with increasing energy depositions which is an expected behavior. However, some contributions are visible with large energy deposition but with smaller TOT values; this can be interpreted as wrongly tagged photons due to accidental coincidences, i.e., instead of $1275 \mathrm{keV}$ photon, photons with lower incident energies were used to estimate the energy deposition for the scattering angles.(e.g., see the Fig. 8c). The accidental coincidence rate for the presented analysis is estimated $\approx 5 \%$. The black line in Fig 11 a is plotted by assuming that the wrongly tagged photons are of energy $511 \mathrm{keV}$. For the final relationship, the profile histograms of Fig. 11a for the most populated energy bins are selected. The TOT spectra for selected energy intervals fitted for a fixed range around the maximum of distribution. The mean values of the TOT distributions as a function of the center value of the energy interval are shown 


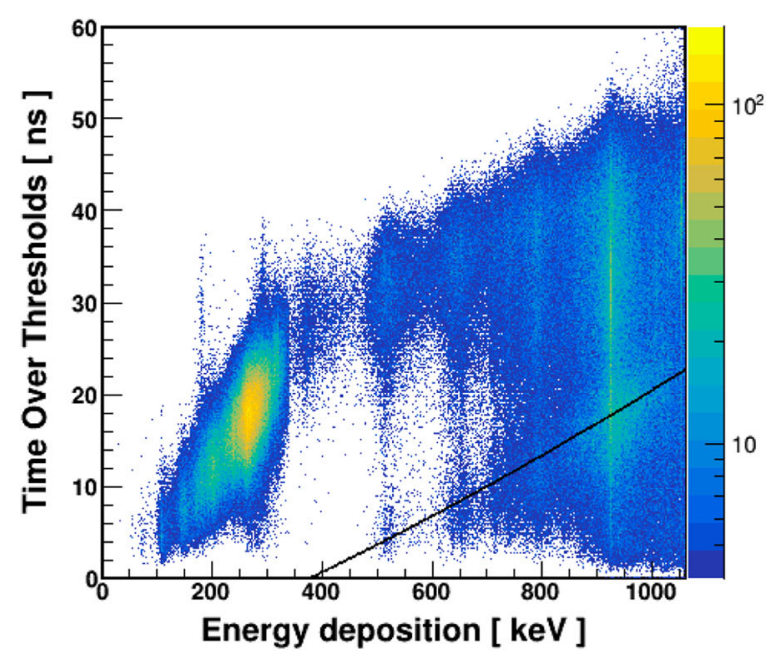

(a)

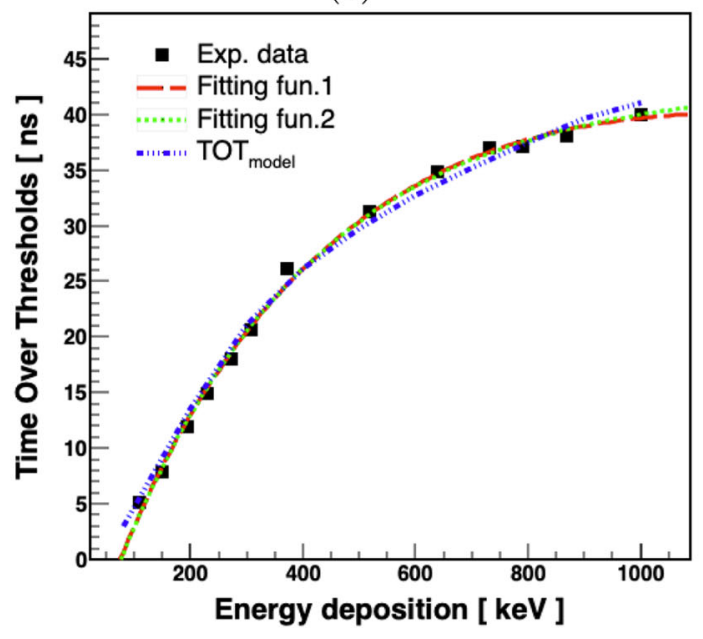

(b)

Fig. 11 a 2-D spectrum of TOT versus energy deposition. b TOT vs energy deposition. Black rectangles correspond to the experimental data. The statistical errors in measuring the values are smaller than the size of symbols due to the large number of entries for the fitted TOT distributions. The red dashed line indicates result of the fit of the function: TOT $=A 0+A 1 * \ln \left(E_{d e p}+A 2\right)+A 3 *\left(\ln \left(E_{d e p}+A 2\right)\right)^{2}$, with $A 0=-2322 n s, A 1=$ $632.1 \mathrm{~ns} / \mathrm{keV}, \mathrm{A} 2=590.2 \mathrm{keV}$, and $\mathrm{A} 3=-42.29 \mathrm{~ns} /(\mathrm{keV})^{2}$. Green dotted line shows the result of another fitting function TOT $=\mathrm{A} 0 \mathrm{~ns}-\mathrm{A} 1{ }^{*} \mathrm{~A} 2^{E_{\text {dep }}}$ with three parameters where $\mathrm{A} 0=42.96 \mathrm{~ns}, \mathrm{~A} 1=53.43 \mathrm{~ns}$, and $\mathrm{A} 2=$ $0.997 \mathrm{keV}^{-1}$. Blue dotted-dashed line represents the model predictions (the "Theoretical model" section) for the total TOT values at four fixed thresholds for the time distribution spectra calculated by using Eq. 3. In framework of J-PET, the light signals are collected on both sides of plastic scintillator as a measure of energy deposition, so in calculating the TOT model, we used twice the value of TOT sum $_{\text {and }}$ and the value of free parameter $N_{0}$ is 1.3

in Fig. 11b. The black rectangles are the experimental data. Errors in estimating the mean value of the TOT are within the size of the used symbols. It is shown that the used functions (see the caption of Fig. 11) are able to reproduce the data for quite a large range of energy deposition, i.e., up to $1000 \mathrm{keV}$. The experimental data is confronted also with a theoretical model (the "Theoretical model" section) which well describes the data.

\section{Conclusions}

J-PET is the first PET scanner composed of plastic scintillators [3, 30]. Plastic scintillators are more than an order of magnitude less expensive than crystal scintillators. The 
time-over-threshold approach facilitates a compact, fast, and cost-effective signal readout $[32,42,43]$. In the framework of J-PET scanner, we adopt the TOT approach as a measure of the energy deposition in order to utilize the fast timing and low pile-up features of the plastics scintillators. To apply this method effectively, in comparison to the classical method of charge collection, it is important to precisely describe the non-linear relationship between the energy deposition of an initial photon and the measured TOT values. Here, we presented a method for determining this relation which we showed can be efficiently applied to the J-PET tomograph by collecting the data with a ${ }^{22} \mathrm{Na}$ source covered with the porous material characterized with the long lifetime of positronium atoms [29]. The identification of an incident photon was based on the angular correlation between the three hits and the lifetime of the metastable positronium atoms while the scattered photon was identified and associated to the initial photon based on the correlation among the registration time and distance between the interaction points. Using a ${ }^{22} \mathrm{Na}$ source emitting $1275 \mathrm{keV}$ prompt and $511 \mathrm{keV}$ annihilation photons, the TOT versus energy loss relationship up to about $1000 \mathrm{keV}$ was established. The proposed functions fits the experimental data well and can be used as a standalone functions for the energy loss calibration of the plastic scintillators used in the J-PET scanner. Furthermore, we also introduced a theoretical model following a simple algorithm able to reproduce the experimental data which may be used in other experimental facilities using the plastic scintillators.

Acknowledgements

The authors acknowledge technical and administrative support of A. Heczko, M. Kajetanowicz, and W. Migdał.

\title{
Authors' contributions
}

All authors contributed in the elaboration of methods for data analysis, interpretation of results, experimental set-up and data collection, correction of manuscript, and approval. The analysis algorithm was mainly developed by SS along with the PM. SS and PM edited the manuscript. All authors read and approved the final manuscript.

\section{Funding}

This work was supported by The Polish National Center for Research and Development through grant INNOTECH-K1/IN1/64/159174/NCBR/12, the Foundation for Polish Science through the MPD and TEAM PIOR.04.04.00-00-4204/17, the National Science Centre of Poland through grants no. 2016/21/B/ST2/01222, 2017/25/N/NZ1/00861, the Ministry for Science and Higher Education through grants no. 6673/IA/SP/2016, 7150/E338/SPUB/2017/1, 7150/E-338/M/2017, 7150/E-338/M/2018, and the Austrian Science Fund FWF-P26783-N27.

Availability of data and materials

The data that support the findings of this study are available from the corresponding author upon request.

Ethics approval and consent to participate

Not applicable

Consent for publication

Not applicable

Competing interests

The authors declare that they have no competing interests

\begin{abstract}
Author details
${ }^{1}$ Faculty of Physics, Astronomy and Applied Computer Science, Jagiellonian University, prof. Stanisława Łojasiewicza 11, 30-348 Cracow, Poland. ${ }^{2}$ INFN, Laboratori Nazionali di Frascati, 00044 Frascati, Italy. ${ }^{3}$ Institute of Nuclear Physics PAN, Cracow, Poland. ${ }^{4}$ Institute of Physics, Maria Curie-Skł odowska University, 20-031 Lublin, Poland. ${ }^{7}$ Faculty of Physics, University of Vienna, 1090 Vienna, Austria. ${ }^{8}$ Department of Complex Systems, National Centre for Nuclear Research, 05-400 Otwock-Świerk, Poland. ${ }^{9}$ High Energy Physics Division, National Centre for Nuclear Research, 05-400

Otwock-Świerk, Poland. ${ }^{10}$ 2nd Department of General Surgery, Jagiellonian University Medical College, Cracow, Poland.

${ }^{11}$ Department of Physics, College of Education for Pure Sciences, University of Mosul, Mosul, Iraq.
\end{abstract}

Received: 19 November 2019 Accepted: 17 May 2020

Published online: 05 June 2020

References

1. Nygren DR. Converting vice to virtue: can time-walk be used as a measure of deposited charge in silicon detectors?. Intern LBL Note. 1991. 
2. Kipnis I, Collins T, DeWitt J, Dow S, Frey A, Grillo A, et al. A time-over-threshold machine: the readout integrated circuit for the BABAR Silicon Vertex Tracker. IEEE Trans Nucl Sci. 1997;44(3 PART 1):289-97.

3. Moskal $\mathrm{P}$, Rundel $\mathrm{O}$, Alfs $\mathrm{D}$, Bednarski T, Białas $\mathrm{P}$, Czerwiński E, et al. Time resolution of the plastic scintillator strips with matrix photomultiplier readout for J-PET tomograph. Phys Med Biol. 2016;61(5):2025-47.

4. Moskal P, Krawczyk N, Hiesmayr BC, Bala M, Curceanu C, Czerwinski E, et al. Feasibility studies of the polarization of photons beyond the optical wavelength regime with the J-PET detector. Eur Phys J C. 2018;78:970.

5. Saint Gobain Crystals. https://www.crystals.saint-gobain.com/.

6. Wieczorek A, Dulski AK, Niedzwiecki S, Alfs D, Bialas P, Curceanu C, et al. Novel scintillating material 2-(4-styrylphenyl)benzoxazole for the fully digital and MRI compatible J-PET tomograph based on plastic scintillators. PLos One. 2017;12(11):e0186728.

7. Vilardi I, Braem A, Chesi E, Ciocia F, Colonna N, Corsi F, et al. Optimization of the effective light attenuation length of YAP:Ce and LYSO:Ce crystals for a novel geometrical PET concept. Nucl Instr Meth A. 2006;564:506-14.

8. Mao R, Zhang L, Ren-Yuan Z. Optical and scintillation properties of inorganic scintillators in high energy physics. IEEE Trans Nucl Sci. 2008;55:2425-31.

9. Lanthanum Bromide Properties. https://www.crystals.saint-gobain.com/products/standard-and-enhancedlanthanum-bromide.

10. Olcott PD, Levin CS. Pulse width modulation: a novel readout scheme for high energy photon detection. IEEE Nucl Sci Symp Conf Rec. 2008. https://doi.org/10.1109/nssmic.2008.4774297.

11. Fujiwara T, Takahashi H, Shimazoe K, Shi B. Multi-level time-over-threshold method for energy resolving multi-channel systems. IEEE Trans Nucl Sci. 2010;57(5):2545-8. https://doi.org/10.1109/tns.2010.2061236.

12. Orita T, Koyama A, Yoshino M, Kamada K, Yoshikawa A, Shimazoe K, et al. The current mode time-over-threshold ASIC for a MPPC module in a TOF-PET system. Nucl Inst Methods Phys Res Sect A Accelerators Spectrometers Detectors Associated Equipment. 2018;912:303-8.

13. Kim H, Kao CM, Xie $\mathrm{Q}$, Chen $C T$, Zhou L, Tang F, et al. A multi-threshold sampling method for TOF-PET signal processing. Nuclear Instruments and Methods in Physics Research, Section A: Accelerators, Spectrometers, Detectors and Associated Equipment. 2009;602(2):618-21.

14. Grant AM, Levin CS. A new dual threshold time-over-threshold circuit for fast timing in PET. Phys Med Biol. 2014;59(13):3421-30.

15. Shimazoe K, Takahashi H, Shi B, Orita T, Furumiya T, Ooi J, et al. Dynamic time over threshold method. IEEE Trans Nucl Sci. 2012;59(6):3213-7.

16. Orita T, Shimazoe K, Takahashi H. The dynamic time-over-threshold method for multi-channel APD based gamma-ray detectors. Nucl Inst Methods Phys Res Sect A Accelerators Spectrometers Detectors Associated Equipment. 2015;775:154-61.

17. Vandenberghe S, Mikhaylova E, D'Hoe E, Mollet P, Karp JS. Recent developments in time-of-flight PET. EJNMMI Phys. 2016;3:1-30.

18. Slomka PJ, Pan T, Germano G. Recent advances and future progress in PET instrumentation. Semin Nucl Med. 2016:46:5-19.

19. Vandenberghe S, Moskal P, Karp JS. State of the art in total body PET. EJNMMI Phys. 2020;7:35.

20. Jin-Jie W, Yue-Kun H, Zhi-Jia S, Chong W, Yu-Da Z, Gui-An Y, et al. A study of time over threshold (TOT) technique for plastic scintillator counter. Chin Physics C. 2008;32(3):186-90.

21. Ashrafi S, Ghahremani GM. Energy calibration of thin plastic scintillators using Compton scattered $\gamma$ rays. Nucl Inst Methods Phys Res Sect A Accelerators Spectrometers Detectors Associated Equipment. 2011;642(1):70-4.

22. Kowalski P, Wiślicki W, Raczyński L, Alfs D, Bednarski T, Białas P, et al. Scatter fraction of the J-PET tomography scanner. Acta Physica Polonica B. 2016;47:549-60.

23. Kowalski P, Wilicki W, Shopa RY, Raczyński L, Klimaszewski K, Curceanu C, et al. Estimating the NEMA characteristics of the J-PET tomograph using the GATE package. Phys Med Biol. 2018;63(16):165008-165025.

24. Compton HA. The spectrum of scatterd $x$-rays. Phys Rev. 1923;22(20):2-6.

25. Garwin RL. Thermalization of positrons in metals. Phys Rev. 1953;91:1571-2.

26. Moskal P, Kisielewska D, Curceanu C, Czerwiński E, Dulski K, Gajos A, et al. Feasibility study of the positronium imaging with the J-PET tomograph. Phys Med Biol. 2019;64(5):055017.

27. Moskal P, Jasińska B, Stępień E. Ł., Bass S. Positronium in Medicine and Biology. Nat Rev Phys. 2019;1:527-9. https:// doi.org/10.1038/s42254-019-0078-7.

28. Dulski K, Curceanu C, Czerwiński E, Gajos A. Commissioning of the J-PET detector in view of the positron annihilation lifetime spectroscopy. Hyperfine Interactions. 2018;239(40):1-6.

29. Jasińska B, Gorgol M, Wiertel M, Zaleski R, Alfs D, Bednarski T, et al. Determination of the $3 \gamma$ fraction from positron annihilation in mesoporous materials for symmetry violation experiment with J-PET scanner. Acta Physica Polonica B. 2016;47(2):453-60

30. Niedźwiecki S, Białas P, Curceanu C, Czerwiński E, Dulski K, Gajos A, et al. J-PET: a new technology for the whole-body PET imaging. Acta Phys Polon B. 2017:48(10):1567-76.

31. Raczyński L, Moskal P, Kowalski P, Wiślicki W, Bednarski T, Bia P, et al. Compressive sensing of signals generated in plastic scintillators in a novel J-PET instrument. Nucl Inst Methods Phys Res Sect A Accelerators Spectrometers Detectors Associated Equipment. 2015;786:105-12.

32. Pałka M, Strzempek P, Korcyl G, Bednarski T, Niedźwiecki S, Białas P, et al. Multichannel FPGA based MVT system for high precision time (20 ps RMS) and charge measurement. J Instrum. 2017;12:P08001.

33. Raczyński L, Moskal P, Kowalski P, Wiślicki W, Bednarski T, Białas P, et al. Novel method for hit-position reconstruction using voltage signals in plastic scintillators and its application to positron emission tomography. Nucl Inst Methods Phys Res Sect A Accelerators Spectrometers Detectors Associated Equipment. 2014;764:186-92.

34. Moskal P, Niedźwiecki S, Bednarski T, Czerwiński E, Kapłon, Kubicz E, et al. Test of a single module of the J-PET scanner based on plastic scintillators. Nucl Inst Methods Phys Res Sect A Accelerators Spectrometers Detectors Associated Equipment. 2014;764:317-21. 
35. Sharma NG, Silarski M, Bednarski T, Bialas P, Czerwiński E, Gajos A, et al. Reconstruction of hit time and hit position of annihilation quanta in the J-PET detector using the Mahalanobis distance. Nukleonika. 2015;60(4):765-9.

36. Won JY, Lee JS. Highly integrated FPGA-only signal digitization method using single-ended memory interface input receivers for time-of-flight PET detectors. IEEE Trans Biomed Circ Syst. 2018;2865581:1-10.

37. Won JY, Kwon SI, Yoon HS, Ko GB, Son JW, Lee JS. Dual-phase tapped-delay-line time-to-digital converter with on-the-fly calibration implemented in 40 nm FPGA. IEEE Trans Biomed Circ Syst. 2016;10:231-42.

38. Won JY, Lee JS. Time-to-digital converter using a tuned-delay line evaluated in 28-, 40-, and 45-nm FPGAs. IEEE Trans Instrum Meas. 2016;65:1678-89.

39. Pałka M, Moskal P, Bednarski T, Białas P, Czerwiński E, Kapłon $Ł$, et al. A novel method based solely on field programmable gate array (FPGA) units enabling measurement of time and charge of analog signals in positron emission tomography (PET). BioAlgoritm MedSyst. 2014;10:41-5.

40. Kim H, Kao CM, Xie Q, Chen CT, Zhou L, Tang F, et al. A multi-threshold sampling method for TOF-PET signal processing. Nucl Instr and Meth A. 2009;602:618-21.

41. Xi D, Zheng C, Liu W, Liu X, Wan L, Kim H, et al. A PET detector module using FPGA-only MVT digitizers. Nucl Sci Symp Med Imaging Conf (NSS/MIC) IEEE. 2013;10:1-5.

42. Korcyl G, Alfs D, Bednarski T, Białas P, Czerwiński E, Dulski K, et al. Sampling FEE and trigger-less DAQ for the J-PET scanner. Acta Phys Pol B. 2016;47(2):491-6.

43. Korcyl G, Białas P, Curceanu C, Czerwi E, Hiesmayr BC, Jasi B, et al. Evaluation of single-chip, real-time tomographic data processing on FPGA SoC devices. IEEE Trans Med Imaging. 2018;37(11):2526-35.

44. Pawlik-Niedźwiecka M, Niedźwiecki S, Białas P, Curceanu C, Czerwiński E, Dulski K, et al. Preliminary studies of J-PET detector spatial resolution. Acta Phys Polon A. 2017;132(5):1645-8.

45. Lecoq $P$, Auffray E, Brunner $S$, Hillemanns $H$, Jarron $P$, Knapitsch $A$, et al. Factors influencing time resolution of scintillators and ways to improve them. IEEE Trans Nucl Sci. 2010;57(5):2411-6.

46. Krzemień W, Bala M, Bednarski T, Bialas P, Czerwiński E, Gajos A, et al. Processing optimization with parallel computing for the J-PET scanner. Nukleonika. 2015;60(4):745-8.

47. Krzemień W, Alfs D, Białas P, Czerwiński E, Gajos A, Głowacz B, et al. Overview of the software architecture and data flow for the J-PET tomography device. Acta Phys Polon B. 2016;47(2):561-7.

48. Kamińska D, Gajos A, Czerwiński E, Alfs D, Bednarski T, Białas P, et al. A feasibility study of ortho-positronium decays measurement with the J-PET scanner based on plastic scintillators. Eur Phys J C. 2016;76(445):1-14.

49. Czerwiński E, Dulski K, Białas P, Curceanu C, Czerwiński E, Dulski K, et al. Commissioning of the J-PET detector for studies of decays of positronium atoms. Acta Phys Polon B. 2017;48(10):1961-8.

50. Moszyński M, Bengtson B. Light pulse shapes from plastic scintillators. Nucl Inst Methods. 1977;142:417-34.

51. Moszyński M, Bengtson B. Status of timing with plastic scintillation detectors. Nucl Inst Methods. 1979;158:1-31.

\section{Publisher's Note}

Springer Nature remains neutral with regard to jurisdictional claims in published maps and institutional affiliations.

\section{Submit your manuscript to a SpringerOpen ${ }^{\circ}$ journal and benefit from:}

- Convenient online submission

- Rigorous peer review

- Open access: articles freely available online

- High visibility within the field

- Retaining the copyright to your article

Submit your next manuscript at $\boldsymbol{\triangleright}$ springeropen.com 